\title{
Optimizing the Power Output for a Capacitive Wireless Power Transfer System with $N$ receivers
}

\author{
Ben Minnaert*, Franco Mastri ${ }^{\dagger}$, Alessandra Costanzo ${ }^{\dagger}$, Mauro Mongiardo ${ }^{\ddagger}$ and Nobby Stevens ${ }^{\S}$ \\ ${ }^{*}$ Odisee University College of Applied Sciences, Ghent, Belgium, Email: ben.minnaert@odisee.be \\ ${ }^{\dagger}$ Dep. of Electrical, Electronic and Information Engineering Guglielmo Marconi, University of Bologna \\ Bologna, Italy, Email: franco.mastri@unibo.it, alessandra.costanzo@unibo.it \\ ${ }^{\ddagger}$ Dep. of Engineering, University of Perugia, Perugia, Italy, Email: mauro.mongiardo@unipg.it

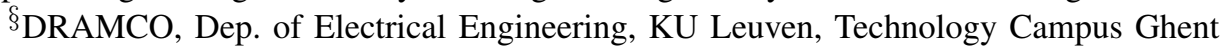 \\ Ghent, Belgium, Email: nobby.stevens@kuleuven.be
}

\begin{abstract}
Capacitive wireless power transfer from one transmitter to multiple receivers is discussed and analytically solved for the realization of maximum power transfer to uncoupled receivers. Simple closed-form expressions for the optimal terminating loads and the corresponding power output and transducer gain are determined by applying the maximum power transfer theorem. The results are validated by circuital simulation.

Index Terms-capacitive wireless power transfer, resonance, wireless power transfer, multiple receivers, maximum power transfer theorem.
\end{abstract}

\section{INTRODUCTION}

Capacitive wireless power transfer (CWPT) uses the electric field as medium to transfer energy from a conducting transmitter plate to a receiver plate. An alternating voltage on the transmitter plate generates an electric field. By capacitive (also called electric) coupling, other plates can receive the transmitted energy for the generation of current in a load circuit. In this way, energy can be transferred wirelessly.

Most of the studies reported in literature focus on a CWPT system with a single transmitter and a single receiver [1]. But since the plates of the wireless link are cheap and simple, CWPT allows for a power transfer system with a single large transmitter plate and multiple smaller receiver plates (Fig. 1). A single transmitter can charge multiple receivers at once.

A wireless power transfer system is typically designed for one of two optimal configurations, depending on the application:

- For the first configuration, the goal is to maximize the amount of power transferred from the transmitter to the receiver(s), which is an asset for e.g., the wireless charging of biomedical implants.

- The goal of the second configuration is to optimize the efficiency of the total system, i.e., to maximize the ratio of the total output power to the input power. In particular for high power applications, e.g., the wireless charging of electric vehicles, this configuration is preferred.

It is important to note that both configurations require different set-ups. Different loads at the receiver's end will result in varying amount of power transferred or varying efficiency. Maximizing the amount of power transferred usually does not maximize efficiency, and vice versa.

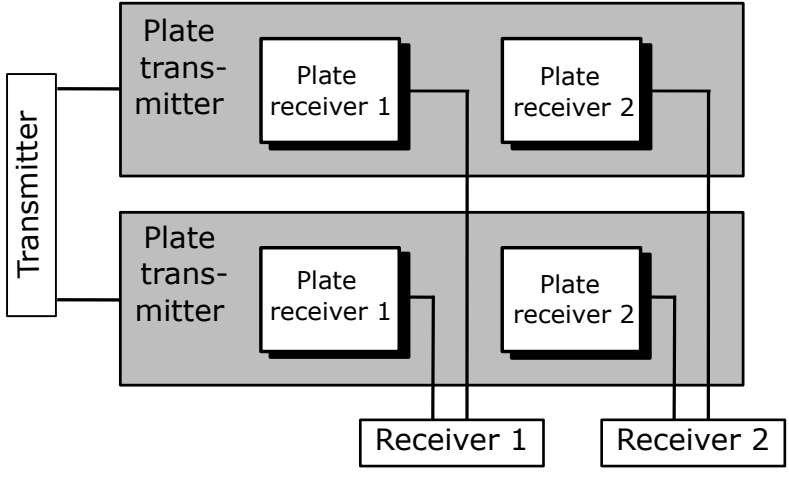

Fig. 1. Example configuration of the transmitter and receiver plates of a capacitive wireless link with one transmitter and two receivers.

In this work, only the first configuration is studied: the optimal solution for maximizing the power transfer for a general CWPT system with one transmitter and multiple receivers is analytically determined. This was already done for a system with a single receiver [2] or two receivers [3], but now the work is extended to $N$ receivers. Closed-form expressions for the optimal terminating loads and the corresponding maximum power transfer are determined from general maximum power transfer theories [4], [5] and applied on the case of capacitive charging. The transducer gain of the system, which is a measure for the transferred power, is determined. Moreover, the input power and efficiency of the maximum power transfer configuration for $N$ receivers is given.

\section{Methodology}

\section{A. Equivalent circuit}

A basic capacitive wireless power transfer (CWPT) system with one transmitter and $N$ receivers is considered. The focus is on the wireless link itself and abstraction is made of the remote electronics. Fig. 2 shows the equivalent circuit of the CWPT system. On the left, the transmitter is shown (subscripts 0). Based on Norton's theorem, the supply of the transmitter is depicted with a time-harmonic current source $I_{0}$ with angular frequency $\omega_{0}$. On the right of Fig. 2, the $N$ receivers are shown (subscripts 1 to $N$ ). The admittances of 


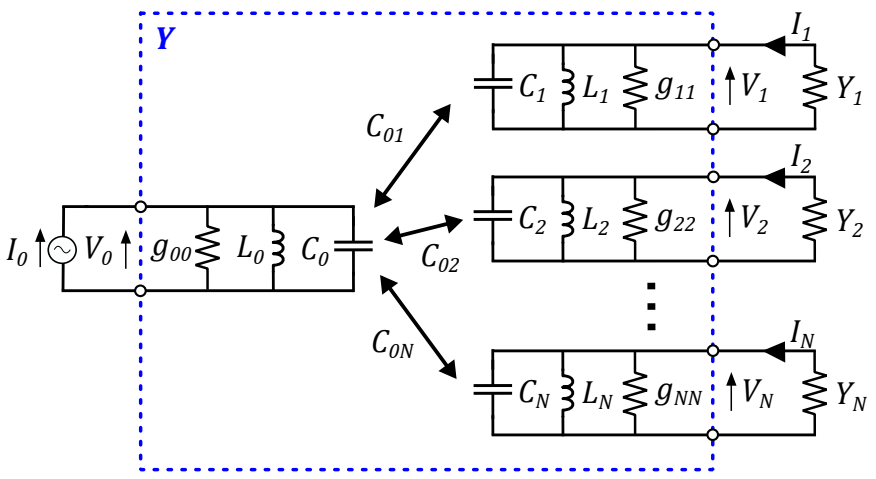

Fig. 2. Equivalent circuit of a capacitive wireless power transfer system with one transmitter (left) and $N$ receivers (right).

the receivers' loads are represented by $Y_{1}$ to $Y_{N}$. The different losses in the circuit are given by the parallel conductances $g_{00}$, $g_{11}, \ldots, g_{N N}$.

The capacitive wireless link itself is described by the coupling between the transmitter capacitance $C_{0}$ and the receivers' capacitances $C_{n}$, given by their mutual capacitance $C_{0 n}$, with $n=1, \ldots, N$.

Resonant circuits are constructed by adding a shunt inductor $L_{i}$ to each circuit, given by $(i=0, \ldots, N)$ :

$$
L_{i}=\frac{1}{\omega_{0}^{2} C_{i}}
$$

Note that this configuration is not the physical structure of a CWPT system, but an approximate equivalent circuit representation thereof [6]-[8]. A more accurate equivalent circuit representation would include series resistances for the inductor losses. However, our approximation allows for a lucid analytical model with the goal of providing a first order estimation of a CWPT system with multiple receivers.

It is important to note that also a coupling can be present between the capacitances of the receivers, e.g., a non-zero mutual capacitance $C_{12}$ between the first and the second receiver. In this work, uncoupled receivers are considered, since in most practical CWPT systems the receivers' coupling can be neglected.

The coupling factor $k_{0 n}$ between the transmitter and each receiver is defined as:

$$
k_{0 n}=\frac{C_{0 n}}{\sqrt{C_{0} C_{n}}}
$$

The circuit in Fig. 2 can be considered as an $(N+1)$-port network with at the ports peak voltage phasors $V_{i}$ and peak current phasors $I_{i}$, as defined in the figure. With Kirchhoff's current laws, the relations between the voltages and currents of the $(N+1)$-port network are obtained:

$$
\boldsymbol{I}=\boldsymbol{Y} \cdot \boldsymbol{V}
$$

The $(N+1) \times 1$ matrices $\boldsymbol{V}$ and $\boldsymbol{I}$ are defined as

$$
\boldsymbol{V}=\left[\begin{array}{c}
V_{0} \\
V_{1} \\
V_{2} \\
\vdots \\
V_{N}
\end{array}\right], \boldsymbol{I}=\left[\begin{array}{c}
I_{0} \\
I_{1} \\
I_{2} \\
\vdots \\
I_{N}
\end{array}\right]
$$

The $(N+1) \times(N+1)$ admittance matrix $\boldsymbol{Y}$ of the $(N+1)$ network is indicated by the dashed rectangle in Fig. 2. At the resonance frequency $\omega_{0}$, the admittance matrix $\boldsymbol{Y}$ is given by

$$
\boldsymbol{Y}=\left[\begin{array}{ccccc}
g_{00} & -j b_{01} & -j b_{02} & \ldots & -j b_{0 N} \\
-j b_{01} & g_{11} & 0 & \ldots & 0 \\
-j b_{02} & 0 & g_{22} & \ldots & 0 \\
\vdots & \vdots & \vdots & \ddots & \vdots \\
-j b_{0 N} & 0 & 0 & \ldots & g_{N N}
\end{array}\right]
$$

with $b_{0 n}=\omega_{0} C_{0 n}$.

\section{B. Maximum power output}

$P_{n}$ is called the power dissipated in load $Y_{n}$. The total power output of the system $P_{\text {out }}$ is defined as

$$
P_{\text {out }}=\sum_{k=1}^{N} P_{k}
$$

The 'available power of the generator' $P_{\max }$ is given by [9]:

$$
P_{\max }=\frac{\left|I_{0}\right|^{2}}{8 g_{00}}
$$

The transducer gain $G_{T}$ equals the ratio between the power dissipated by all the loads and the available power of the generator:

$$
G_{T}=\frac{P_{\text {out }}}{P_{\max }}
$$

Maximizing $G_{T}$ corresponds to maximizing the amount of power transferred to the loads.

By applying the maximum power transfer theorem for $N$ port networks [4], [5], the set of optimal loads $Y_{n}=G_{n}+j B_{n}$ can be determined that maximize $G_{T}$ of the system, with $G_{n}$ and $B_{n}$ the load conductance and susceptance, respectively.

From Norton's theorem, the network represented by $\boldsymbol{Y}$ can be substituted to the equivalent Norton network with admittance matrix:

$$
\boldsymbol{Y}_{\mathbf{0}}=\left[\begin{array}{cccc}
g_{11}+\frac{b_{01}^{2}}{g_{00}} & \frac{b_{01} b_{02}}{g_{00}} & \cdots & \frac{b_{01} b_{0 N}}{g_{00}} \\
\frac{b_{02} b_{01}}{g_{00}} & g_{22}+\frac{b_{02}^{2}}{g_{00}} & \cdots & \frac{b_{02} b_{0 N}}{g_{00}} \\
\vdots & \vdots & \ddots & \vdots \\
\frac{b_{0 N} b_{01}}{g_{00}} & \frac{b_{0 N} b_{02}}{g_{00}} & \cdots & g_{N N}+\frac{b_{0 N}^{2}}{g_{00}}
\end{array}\right]
$$


and $N$ current sources $J_{i}(i=1, \ldots, N)$ given by:

$$
J_{i}=j I_{0} \frac{b_{0 i}}{g_{00}}
$$

The $(N \times 1)$-matrix $\boldsymbol{J}_{0}$ is defined as:

$$
\boldsymbol{J}_{\mathbf{0}}=\left[\begin{array}{c}
J_{1} \\
J_{2} \\
\vdots \\
J_{N}
\end{array}\right]
$$

Based upon [4], [5], maximum power transfer is realized if the voltage matrix $V$ is equal to:

$$
\boldsymbol{V}=\boldsymbol{V}_{\boldsymbol{m a x}}=\left(\boldsymbol{Y}_{\mathbf{0}}+\boldsymbol{Y}_{\mathbf{0}}^{*}\right)^{-1} \boldsymbol{J}_{\mathbf{0}}=\frac{j I_{0}}{2 g_{00} \theta_{N}^{2}}\left[\begin{array}{c}
\frac{b_{01}}{g_{11}} \\
\frac{b_{02}}{g_{22}} \\
\vdots \\
\frac{b_{0 N}}{g_{N N}}
\end{array}\right]
$$

with $\boldsymbol{Y}_{\mathbf{0}}^{*}$ the conjugate transpose of $\boldsymbol{Y}_{\mathbf{0}}$ and

$$
\theta_{N}^{2}=1+\frac{1}{g_{00}} \sum_{k=1}^{N} \frac{b_{0 k}^{2}}{g_{k k}}
$$

The optimal loads $Y_{n}=G_{n}+j B_{n}$ that realize maximum power transfer can be found from the expression [4]

$$
\operatorname{diag}\left(Y_{1}, Y_{2}, \ldots, Y_{N}\right) \cdot \boldsymbol{V}_{\boldsymbol{m a x}}=\boldsymbol{Y}_{\mathbf{0}}^{*} \boldsymbol{V}_{\boldsymbol{m a x}}=\frac{j I_{0}}{2 g_{00}}\left[\begin{array}{c}
b_{01} \\
b_{02} \\
\vdots \\
b_{0 N}
\end{array}\right]
$$

A purely real admittance is found:

$$
\left\{\begin{array}{l}
G_{n}=g_{n n} \theta_{N}^{2} \\
B_{n}=0
\end{array}\right.
$$

The optimal loads are purely resistive because no coupling between the receivers is present [5].

The maximum output power $P_{\text {out }, \text { max }}$ is given by [4]

$$
P_{\text {out }, \max }=\frac{1}{4} \boldsymbol{J}_{\mathbf{0}}^{*} \boldsymbol{V}_{\max }
$$

Combining the above expression with (7) and (8), the transducer gain $G_{T}$ can be determined:

$$
G_{T}=\frac{\sum_{k=1}^{N} \chi_{0 k}^{2}}{\theta_{N}^{2}}
$$

with

$$
\chi_{0 n}^{2}=\frac{b_{0 n}^{2}}{g_{00} g_{n n}}
$$

The normalized input power $p_{i n}$ of the CWPT system, normalized to $P_{\max }$, for the maximum power transfer configuration is given by:

$$
p_{\text {in }}=2 \frac{1+\theta_{N}^{2}}{\theta_{N}^{2}}
$$

The efficiency $\eta$ of the system, defined as the ratio of the output power to the input power of the system, at the maximum power transfer configuration, is given by:

$$
\eta=\frac{\theta_{N}^{2}-1}{2\left(1+\theta_{N}^{2}\right)}
$$

In the next section, the above analytical derivation is verified by simulating a CWPT system consisting of one transmitter and three receivers with different couplings.

\section{NUMERICAL VERIFICATION}

If a single $(N=1)$ or two $(N=2)$ receivers are present, the analytical expressions are reduced to the same expressions found in [2] and [3], respectively, for a CWPT system with one transmitter and a single or two uncoupled receivers.

The above analytical calculation is validated by numerical circuital simulation. Consider the set-up of Fig. 2 with one transmitter and three receivers, which are mutually uncoupled. Typical values for the system are chosen, given in Table I, which are representative values for the following set-up: a transmitter consisting of a large aluminium plate; different receiver aluminium plates of sizes $10 \mathrm{~cm} \times 10 \mathrm{~cm}$, coated with polyethylene as a dielectric material; distances of 2 to $3 \mathrm{~mm}$ between transmitter and receiver [6], [8].

TABLE I

THE FOLLOWING SIMULATION PARAMETERS ARE CONSIDERED FOR THE CWPT SYSTEM WITH ONE TRANSMITTER AND THREE RECEIVERS.

\begin{tabular}{|c|c||c|c|}
\hline Quantity & Value & Quantity & Value \\
\hline$g_{00}$ & $100 \mathrm{mS}$ & $C_{0}$ & $350 \mathrm{pF}$ \\
$g_{11}$ & $150 \mathrm{mS}$ & $C_{1}$ & $250 \mathrm{pF}$ \\
$g_{22}$ & $175 \mathrm{mS}$ & $C_{2}$ & $225 \mathrm{pF}$ \\
$g_{33}$ & $200 \mathrm{mS}$ & $C_{3}$ & $200 \mathrm{pF}$ \\
$C_{01}$ & $150 \mathrm{pF}$ & $f$ & $10 \mathrm{MHz}$ \\
$C_{02}$ & $100 \mathrm{pF}$ & & \\
$C_{03}$ & $50 \mathrm{pF}$ & & \\
\hline
\end{tabular}

The resonance inductances, coupling factors, and auxiliary parameters are calculated from equations (1), (2), (13) and (18) and are listed in Table II.

TABLE II

Calculated values For THE Simulated CWPT System

\begin{tabular}{|c|c||c|c|}
\hline Quantity & Value & Quantity & Value \\
\hline$L_{0}$ & $0.72 \mu \mathrm{H}$ & $k_{01}$ & $51 \%$ \\
$L_{1}$ & $1.0 \mu \mathrm{H}$ & $k_{02}$ & $36 \%$ \\
$L_{2}$ & $1.1 \mu \mathrm{H}$ & $k_{03}$ & $19 \%$ \\
$L_{3}$ & $1.3 \mu \mathrm{H}$ & $\theta_{N}$ & 9.4 \\
$\chi_{01}$ & 7.7 & & \\
$\chi_{02}$ & 4.8 & & \\
$\chi_{03}$ & 2.2 & & \\
\hline
\end{tabular}

From (15), the load conductances $G_{1, \max }, G_{2, \max }$ and $G_{3, \max }$ are found that realize maximum power transfer to the receivers. The corresponding transducer gain $G_{T}$, normalized input power $p_{i n}$, and efficiency $\eta$ for this maximum power configuration are derived from (8), (19), and (20). The values of the parameters at the maximum power point are given in Table III. 
TABLE III

CALCULATED VALUES FOR THE MAXIMUM POWER CONFIGURATION OF THE CWPT SYSTEM

\begin{tabular}{|c|c||c|c|}
\hline Quantity & Value & Quantity & Value \\
\hline$G_{1, \max }$ & $132 \mathrm{mS}$ & $p_{i n}$ & 2.02 \\
$G_{2, \max }$ & $153 \mathrm{mS}$ & $G_{T}$ & 0.989 \\
$G_{3, \max }$ & $175 \mathrm{mS}$ & $\eta$ & $48.9 \%$ \\
\hline
\end{tabular}

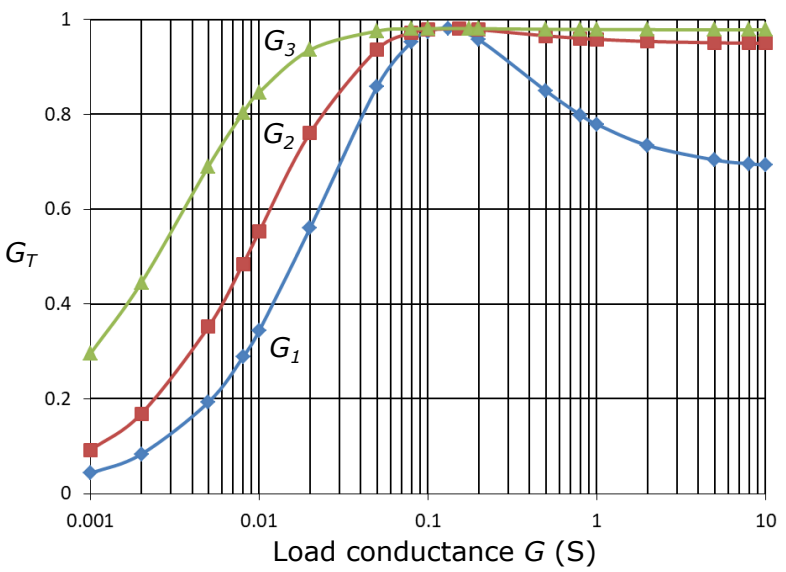

Fig. 3. The simulated transducer gain $G_{T}$ as function of varying load conductances. One load conductance is varied, while keeping the other load conductances at their optimal value for maximum power transfer.

The system is simulated in SPICE for varying load conductances to numerically verify the calculated results. One load conductance is varied, while keeping the other load conductances at their optimal value for maximum power transfer. Figure 3 shows the simulation results: the transducer gain $G_{T}$ as function of varying load conductance. A maximum $G_{T}$ of 0.989 is obtained by the simulation at the load conductances $G_{1}, G_{2}$, and $G_{3}$ of $132 \mathrm{mS}, 153 \mathrm{mS}$, and $175 \mathrm{mS}$, respectively. This is in accordance with the analytical results from Table III. Additionally, the simulated input power and efficiency at this maximum power point correspond with the analytical calculated values.

\section{CONCLUSION}

A general capacitive wireless power transfer system with one transmitter and an arbitrarily number of (uncoupled) receivers was solved for the maximum power transfer configuration. The set-up for obtaining a maximum amount of power transfer from a single transmitter to all receivers was determined, i.e. the optimal receivers' loads. It was found that the optimal loads are purely real. Lucid closed-form expressions were calculated for the transducer gain, input power and efficiency in the maximum power point. The analytical derivation was validated by a circuital simulation for an example capacitive system of one transmitter and three receivers with different coupling factors.

\section{REFERENCES}

[1] Lu, F., Zhang, H. and Mi, C., 2017. A review on the recent development of capacitive wireless power transfer technology. Energies, 10(11), p.1752.

[2] Dionigi, M., Mongiardo, M., Monti, G. and Perfetti, R., 2017. Modelling of wireless power transfer links based on capacitive coupling. International Journal of Numerical Modelling: Electronic Networks, Devices and Fields, 30(3-4), p.e2187.

[3] Minnaert, B. and Stevens, N., 2017. Optimal analytical solution for a capacitive wireless power transfer system with one transmitter and two receivers. Energies, 10(9), p.1444.

[4] Desoer, C., 1973. The maximum power transfer theorem for n-ports. IEEE Transactions on Circuit Theory, 20(3), pp.328-330.

[5] Monti, G., Dionigi, M., Mongiardo, M. and Perfetti, R., 2017. Optimal design of wireless energy transfer to multiple receivers: power maximization. IEEE Transactions on Microwave Theory and Techniques, 65(1), pp.260-269.

[6] Minnaert, B. and Stevens, N., 2017. Conjugate image theory applied on capacitive wireless power transfer. Energies, 10(1), p.46.

[7] Hong, J.S.G. and Lancaster, M.J., 2004. Microstrip filters for RF/microwave applications (Vol. 167). John Wiley \& Sons.

[8] Huang, L. and Hu, A.P., 2015. Defining the mutual coupling of capacitive power transfer for wireless power transfer. Electronics Letters, 51(22), pp.1806-1807.

[9] Montgomery, C.G., Dicke, R.H. and Purcell, E.M. eds., 1987. Principles of microwave circuits (No. 25). Iet. 